\title{
Facility layout design using a multi-objective interactive genetic algorithm to support the DM
}

\author{
Laura García-Hernández, ${ }^{1}$ Antonio Arauzo-Azofra, ${ }^{1}$ \\ Lorenzo Salas-Morera, ${ }^{1}$ Henri Pierreval ${ }^{2}$ and Emilio Corchado ${ }^{3}$
}

(1) Area of Project Engineering, University of Cordoba, Spain

E-mail: ir1gahel@uco.es

(2) LIMOS UMR CNRS 6158 IFMA, Clermont-Ferrand, France

(3) Departamento de Informática y Automática, Universidad de Salamanca, Salamanca, Spain

\begin{abstract}
The unequal area facility layout problem (UA-FLP) has been addressed by many methods. Most of them only take aspects that can be quantified into account. This contribution presents a novel approach, which considers both quantitative aspects and subjective features. To this end, a multi-objective interactive genetic algorithm is proposed with the aim of allowing interaction between the algorithm and the human expert designer, normally called the decision maker (DM) in the field of UA-FLP. The contribution of the DM's knowledge into the approach guides the complex search process, adjusting it to the DM's preferences. The entire population associated to facility layout designs is evaluated by quantitative criteria in combination with an assessment prepared by the DM, who gives a subjective evaluation for a set of representative individuals of the population in each iteration. In order to choose these individuals, a soft computing clustering method is used. Two interesting real-world data sets are analysed to empirically probe the robustness of these models. The first UA-FLP case study describes an ovine slaughterhouse plant and the second, a design for recycling carton plant. Relevant results are obtained, and interesting conclusions are drawn from the application of this novel intelligent framework.
\end{abstract}

Keywords: multi-objective, unequal area facility layout problem, interactive evolutionary computation, facility layout

\section{Introduction}

Over the last years, there has been a growing interest in the need for designing intelligent systems to address the relevant industrial issue of the facility layout problem (FLP) (Kusiak and Heragu, 1987). This problem determines the placement of facilities in a manufacturing plant with the aim of determining the most effective arrangement in accordance with predetermined criteria or objectives, under certain constraints. Where to locate facilities and the efficient design of those facilities are important and fundamental strategic issues facing any manufacturing industry (Singh and Sharma, 2006). Well laid out facilities contribute to the overall efficiency of operations and can reduce between $20 \%$ and $50 \%$ of the total operating costs (Tompkins et al., 2010). There are many kinds of layout problems. A possible classification of these problems is based on the study by Kusiak and Heragu (1987).

The design of production plants involves many decisions related to production technologies, selection of processes and facilities layout that should be taken into account, in an integrated framework from the first steps of the design, in order to have efficient and flexible plants (Askin and Mitwasi, 1992). In this context, aspects such as reduction of energy and resource consumption, and control of emissions, among others, should be considered in the whole product life cycle engineering (Umeda et al., 2012). Furthermore, in each phase of the life cycle, decisions about design, evaluation and selection of an effective layout, and production planning and scheduling have to be taken into consideration in order to adapt the production to a changing environment (Raman et al., 2009). Therefore, different points of view, including building, machinery, material handling and facilities, normally developed by different stakeholders, and with different points of view, have to be combined in a cooperative design (Shariatzadeh et al., 2012).

The novel contribution presented here focuses on the unequal area facility layout problem (UA-FLP) as formulated by Armour and Buffa (1963). In their proposal, they described UA-FLP as a rectangular plant layout that is composed of unequal rectangular facilities that have to be placed effectively in the plant layout.

Generally, the problem of designing a physical layout involves the minimization of the material handling cost as one of the main objectives (Aiello et al., 2012), although other quantitative aspects can be taken into account (e.g. closeness or distance relationships, adjacency requirements and aspect ratio). These quantitative features can be addressed by several optimization approaches. However, Babbar-Sebens and Minsker (2012) established that these approaches may not adequately represent all of the relevant qualitative information that affects a human expert involved in the design phase (e.g. engineers). As a result, qualitative features should be taken into consideration, for instance, facility locations' preferences, distribution of the remaining spaces, relative placement preferences or any other subjective preference that can be considered as important by the human expert, usually called the decision maker (DM) in the field of facility layout design. These qualitative features are complicated to consider by any classical heuristic or metaheuristic optimization model (Brintup et al., 2007). In this 
respect, the participation of the DM is essential in order to include qualitative considerations in the design. Additionally, incorporating the DM's expertise into the algorithm provides several additional advantages, such as including expert knowledge, finding a solution that satisfies the DM although not necessarily an optimal solution (Avigad and Moshaiov, 2009), selecting the best trade-off solution when a conflict among objectives or constraints exists (Jeong and Kim, 2009), helping the algorithm in guiding the search process to DM preferences (Luque et al., 2009), eliminating the need to specify all the required preference information in advance, giving the possibility to the DM to learn about his or her own preferences (Jeong and Kim, 2009), stimulating the DM creativity, (Sato and Hagiwara, 2001), and obtaining original, innovated and practicable solutions.

Several evolutionary computation (EC) approaches have been applied to deal with UA-FLP (Yeh and Wu, 2010; Day and Nandi, 2011). Among these, genetic algorithms (GAs) are commonly used (Holland, 1992; Jackowski and Wozniak, 2010; García-Hernández et al., 2009). Brintup et al. (2007) have highlighted that interactive evolutionary computation (IEC) can greatly contribute the improvement of optimized design by involving DMs in the search for a satisfactory solution. In this sense, interactivity features allow more qualitative considerations, which can be more subjective, to be taken into account. In this contribution in IEC, the fitness function is replaced by a human's DM evaluation (Takagi, 2001).

This research proposes a new approach based on the use of an interactive multi-objective algorithm for addressing the UA-FLP. By means of this new approach, a combination of both quantitative and qualitative (using the expert knowledge) criteria are taken into account to determine an acceptable solution.

The novel suggested approach uses the DM's expert knowledge. This fact allows the DM to interact with the algorithm, guiding the search process and adjusting it to DM preferences through the subjective evaluations of representative solutions, which are different enough and are chosen using a clustering method called c-means clustering method (Bezdek et al., 1984). This soft computing technique allows the same element to belong to more than one group (Corchado et al., 2012).

The remainder of this contribution is organized as follows. Section 2 presents an overview of the UA-FLP. In the following section, the intelligent systems applied in the proposed approach are explained. The problem formulation is given in Section 4. Next, Section 5 details the new proposed approach. Section 6 illustrates the suggested approach through two real case studies. Finally, conclusions and future research lines are stated in Section 7.

\section{Overview of the unequal area facility layout problem}

In order to address the UA-FLP (Armour and Buffa, 1963), different approaches have been considered. Taking into account the exact procedures, Meller et al. (1999) proposed general classes of valid inequalities that are based on an acyclic sub-graph structure; they then incorporated the structures into the branch and bound algorithm. Sherali et al.
(2003) presented an improved mixed-integer programming model that uses a polyhedral outer approximation of the area constraints and branching priorities. Konak et al. (2006) used a mixed-integer programming approach for UA-FLPs where the non-linear facility area constraints are modelled in a continuous plane without using any surrogate constraints.

With respect to meta-heuristic approaches, Tam (1992) developed Layout Optimization using Guillotine-Induced Cut, which applies Simulated Annealing, an attempt to find a better layout by two-way exchanges of branching operators. Scholz et al. (2009) presented a slicing tree-based Tabu Search heuristic for the rectangular, continual plane FLP. They also incorporated the possibility of specifying various requirements regarding shape and dimensions of each individual facility by using bounding curves. Many authors addressed the problem using GAs. For instance, Tate and Smith (1995) proposed a GA for solving UA-FLPs using a dynamic or adaptive penalty function to guide the search into feasible solution regions. $\mathrm{Wu}$ and Appleton (2002) presented a GA method to solve the UA-FLP and aisle structure problems simultaneously. Gomez et al. (2003) focused on a particular case, which involves the explicit consideration of passageways between sections with the possibility of these sections being variable in width. Wang et al. (2005) implemented an analysis of variance of statistics to determine the best site size by GA applied to UA-FLP. Enea et al. (2005) implemented a GA to search for a near optimal solution in a fuzzy context. Moreover, constraints on the aspect ratio of the facilities are taken into account using a penalty function introduced into the fitness function of the GA. Aiello et al. (2006) suggested a multi-objective approach to UA-FLP that uses a GA and Electre method. Norman and Smith (2006) addressed the UA-FLP using a GA that considers uncertainty in material handling costs on a continuous scale by using expected values and standard deviations of product forecasts. Liu and Meller (2007) proposed an approach to solve UA-FLP represented as sequence pairs, by using GA and mixed-integer programming. They used GA to modify the solutions represented as sequence pairs, which have the purpose of eliminating all infeasible binary variables that make large UA-FLP difficult to solve. Komarudin and Wong (2010) applied an Ant System to solve UA-FLP. They proposed an algorithm that uses several types of local searches to improve its search performance. Ulutas and Kulturel-Konak (2012) introduced an artificial immune system-based algorithm to solve the UA-FLP with flexible bay structure. They proposed a clonal selection algorithm with a new encoding model and a novel procedure to cope with dummy departments.

Generally, the existing approaches take into consideration features that can be quantified (GarcíaHernández, 2011). Most of them have solved the problem by considering only the material flow within facilities for their optimization (Armour and Buffa, 1963; Komarudin and Wong, 2010; Norman and Smith, 2006; Scholz et al., 2009; Ulutas and Kulturel-Konak, 2012), while other authors have addressed the UA-FLP by taking into account additional features as distance requirements, adjacency 
requests, aspect ratio factor, area utilization factor and so on. This is the case of Wang et al. (2005), Enea et al. (2005) and Aiello et al. (2006, 2012). However, these approaches may not adequately represent all of the relevant qualitative information that affects a human expert involved in design (Babbar-Sebens and Minsker, 2012). In this way, qualitative features are also important to be taken into consideration. Referring to UA-FLP, GarcíaHernández et al. (2013) proposed an interactive genetic algorithm (IGA) capturing the qualitative preferences that the DM would like to have in the final design. Although this approach allows an improvement, it does not, unfortunately, automatically consider the quantitative performance in the obtained solutions (the quantitative performance is just displayed to the DM, who can consider it in their qualitative evaluation). As a result, it is possible to obtain solutions that are considered satisfactory by the DM; however, they are not feasible because of the cost associated to implement them, taking the quantitative criteria into account. In order to solve this issue, a novel research line is presented in the present contribution. It combines both quantitative and qualitative criteria simultaneously, without losing the effectiveness reached by García-Hernandez et al. (2011).

After analysing the existing approaches to the best of our knowledge, there is no approach that addresses the UA-FLP by taking into account the combination of both quantitative criteria and qualitative features in the layout design (involving the DM to fit the qualitative preferences that they would like to see in the final design).

\section{Intelligent systems}

\subsection{Genetic algorithms}

A GA is an heuristic search procedure, which is based on the natural process of evolution as in biological sciences (Holland, 1992; Mitchell, 1998). This meta-heuristic method is used to generate useful solutions for optimization and search problems, which are inspired by natural evolution issues, such as inheritance, selection, mutation and recombination.

The GAs have been successfully applied in solving a variety of optimization problems, which are difficult to solve, including the travelling salesperson problem, job-shop scheduling problems, vehicle routing problems and airline crew scheduling problems, among others (Chaudhry, 2006).

Briefly, the GA begins by generating a random population of possible candidate solutions. Normally, each population element is a string of chromosomes, which represents a possible solution for the particular problem. The elements of this population are evaluated taking into account a given objective function, called the fitness function. The population elements with higher fitness values have a higher probability of participating in the evolutionary operations (crossover and mutation). Crossover process is carried out by selecting two population elements and then exchanging their genetic information. This process produces new solutions that make up the new population. The mutation operation is realized with a low probability over the individuals of the population, and it randomly alters a gene within the chromosome. Mutation process prevents premature loss of important information. Finally, the GA procedure continues until a satisfactory solution is achieved, or until a determined number of generations are executed.

\subsection{Clustering method}

To avoid tiring the DM with too many individual evaluations and to offer a choice between sufficiently different solutions (at least at the beginning), only a representative subset of the population of solutions is submitted to the DM. A clustering method is used to select these representative solutions, which will be displayed to the DM (MacQueen, 1967; Jain, 2010; Baruque et al., 2011). This groups the population into different categories and chooses the element that will represent each category (He and Tan, 2012).

Because of the number and complexity of the features that define the UA-FLP individuals (in this problem, an individual represents a possible facility layout), it is preferable to allow each element to belong to more than one cluster simultaneously. In this way, it can receive inherited evaluations from more than one representative element, which allows the algorithm to accurately adjust the evaluations of the elements that are near the bounds of the clusters. To meet these requirements, an overlapping method of clustering, such as the fuzzy cmeans clustering algorithm detailed by Bezdek et al. (1984), was selected. This clustering method calculates a number of centroids and then chooses the element nearest to the cluster centroid as the representative element from each cluster. For the remaining elements of the population, the membership value $\left(m_{i j}\right)$ is calculated among the particular individual and each of the representative cluster elements. In this way, given the membership grade of a given element to each of the clusters, this element can be categorized as being in the cluster with the higher membership value.

The c-means clustering method is based on minimizing the following objective equation:

$$
F_{m}=\sum_{i=1}^{c} \sum_{j=1}^{n} m_{i j}^{f}\left\|x_{j}-c_{i}\right\|^{2}
$$

The membership grade of each individual with respect to each representative element of the cluster is calculated by using the following equation:

$$
m_{i j}=\frac{1}{\left.\sum_{k=1}^{c}\left(\frac{\| x_{j}-c_{i} \mid}{\| x_{j}-c_{k} \mid}\right)\right)_{f-1}}
$$

The centroid of each cluster is computed as follows:

$$
c_{i}=\frac{\sum_{j=1}^{n} m_{i j}^{f} x_{j}}{\sum_{j=1}^{n} m_{i j}^{f}}
$$


where $F_{m}$ is the function to minimize, $m_{i j}$ is the membership grade of the individual $j$ in the cluster $i, c$ is the number of clusters, $n$ is the number of individuals, $f$ is the fuzziness parameter, which is any real number greater than $1, x_{j}$ is the individual $j$ of the population, $c_{i}$ is the centroid of the cluster $i$ and $\|*\|$ is any norm expressing the similarity between the element and the centroid of the cluster. In our particular case, the metric used is the Euclidean Squared Distance.

The procedure for applying the clustering method is detailed as follows:

1. $M_{0}=\left[m_{i j}\right]_{0}$. The membership matrix is initialized with uniform random numbers in the range of $[0,1]$ such that the sum of the memberships of an element equals (1).

2. At step $K$, the centroids of the clusters are calculated using equation (3).

3. The matrix $M_{k}$ is updated with the centroid values obtained in step 2, using equation (2).

4. If $\left\|M_{k+1}-M_{k}\right\|<\varepsilon$, or the algorithm reaches the step $k_{\max }$, the process ends. Otherwise, the process continues going to step 2. $\varepsilon$ specifies the minimum improvement required to continue the execution of the algorithm. In our particular case, these values are $\varepsilon=0,001$ and $k_{\text {max }}=25$, taken from Bezdek et al. (1984),

where

$$
|| M_{k+1}-M_{k} \|=\max _{i j}\left|\left[m_{i j}\right]_{k+1}-\left[m_{i j}\right]_{k}\right|
$$

\section{Problem formulation}

The UA-FLP (Armour and Buffa, 1963) considers a rectangular plant with fixed dimensions, $W$ (width) $\times H$ (height), and a set of facilities, each with a required area $\left(A_{i}\right)$, where the sum of the facility areas must be less than or equal to the total plant area; see equation (5). The aim is to allocate the facilities in the plant based on a given optimization criterion, subjected to the non-overlapping restriction of facilities.

$$
\sum_{i}^{n} A_{i}<=W \times H
$$

In a number of problems, the efficiency of solutions can be easily quantified; an example is to minimize the material handling costs. However, in this research, the interest is focused on problems where several quantitative and qualitative considerations have to be taken into account. Therefore, a good solution cannot be easily formulated as an objective function, which is why taking the DM's knowledge into account is essential in the approach.

In order to find a satisfactory solution for the DM, some objectives and constraints can be set up. In the novel method proposed in this study, a good solution is one that minimizes the material handling cost and maximizes DM satisfaction. Additionally, the aspect ratio constraint must be satisfied. All these aspects are explained later.

\subsection{Quantitative objective aspects}

Material handling cost reflects the total cost associated with the flow of material movements that exists in the plant layout. Equation (6) shows how it is calculated:

$$
C=\sum_{i}^{n} \sum_{j}^{n} f_{i j} d_{i j} c_{i j}
$$

where $n$ is the total number of facilities in the plant, $f_{i j}$ is the material flow between the facilities $i$ and $j, d_{i j}$ is the Euclidean distance between $i$ and $j$, and $c_{i j}$ is the cost to move a unit of material flow from the facility $i$ to facility $j$.

\subsection{Qualitative subjective aspects}

The subjective aspects refer to the interests that the DM would prefer in each plant design. For example, the DM's interests can be the following, among others:

1. The way that the remaining space is distributed in the plant layout. In this respect, the DM may want solutions that have, for instance, all the remaining space either concentrated in a determined location or distributed in certain areas of the plant. For example, it can be dispersed throughout the plant layout to be used as storage rooms among facilities.

2. Preferences about placement, which could imply that a specific facility will be placed in the south front, in the centre or in a corner of the plant layout.

3. Facility orientation. This aspect involves the orientation that the DM prefers for a given facility so that it may, for example, better suit the sequence of the productive process.

4. Locations to be avoided. This aspect could be interesting for the DM when undesirable factors (e.g. noise, bad smells and humidity) exist in the plant, and it is necessary to avoid specific locations for certain facilities.

5. Any other subjective interest that the DM would like to consider.

\subsection{Aspect ratio constraint}

Facilities separated from a given area must also have a usable shape that allows the allocation of machines or other resources. This is a constraint that defines a subset of feasible solutions in the search space. An adequate aspect ratio is required for each facility, which is defined as a maximum allowed value for equation (7). This equation divides the highest dimension by the shortest one.

$$
A R_{j}=\frac{\max \left\{h_{j}, w j\right\}}{\min \left\{h_{j}, w j\right\}}
$$

where $h$ and $w$ are height and width dimensions of facility $j$.

\section{Proposed multi-objective interactive genetic algorithm}

The structure of the novel multi-objective interactive genetic algorithm (MO-IGA) presented in this study is illustrated in Figure 1. The steps are the following: 


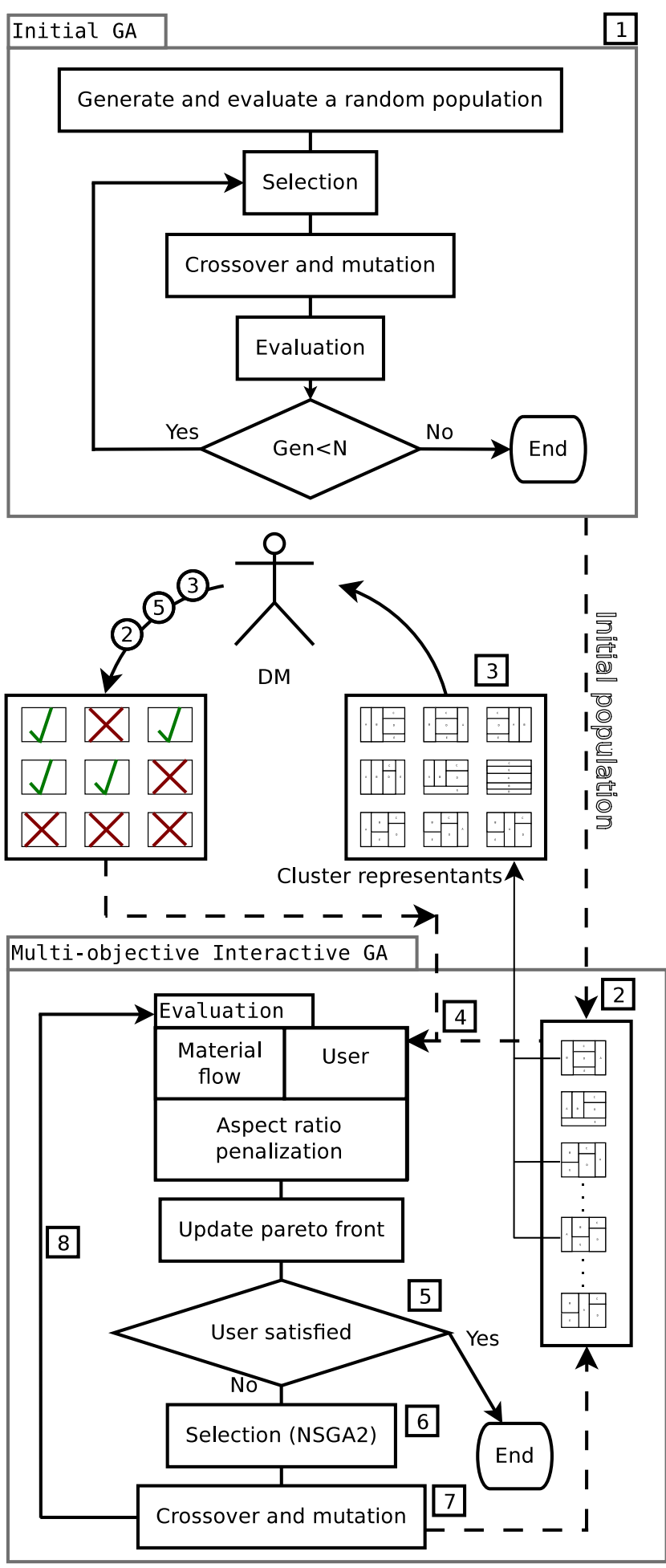

Figure 1: Proposed multi-objective interactive genetic algorithm, based on eight steps.

1. Without disturbing the DM, a non-interactive GA is run to generate an initial population of $N$ individuals. As a result, the IGA starts from a varied subset of solutions that are feasible according to aspect ratio and have good material flow values. In this way, the DM is rarely presented with a plant with poor values for quantitative objectives.

2. A version of the fuzzy c-means clustering algorithm (Bezdek et al., 1984) is applied over the population, grouping the individuals into $c$ clusters (Section 1).
3. A representative element from each of the $c$ clusters is displayed to the DM.

4. Considering the subjective evaluation from the DM to the representative elements of the clusters and taking into account the membership grade of each cluster, the subjective fitness evaluation for each individual is calculated. Material flow is also evaluated, and penalization is applied over both measures. After the whole population has been successfully evaluated, the pareto front with the non-dominated solutions is updated for future reference.

5. If the DM is satisfied with a particular solution, the process successfully ends. Otherwise, the process continues. Additionally, DMs can keep solutions that are especially interesting for them. These solutions are visible for the DM during all the process, so that they can choose a solution that has appeared at any iteration.

6. The selection method is applied to choose the individuals that will carry out the evolutionary operations.

7. Crossover and Mutation operators are applied to the individuals with probabilities given as parameters.

8. The new population is created. The process continues and goes to step 2.

\subsection{Layout representation}

The plant layout design is created using the flexible bay structure (FBS). FBS is currently receiving great attention from researchers (Wong and Komarudin, 2010). The UA-FLP plant is delimited by height $(H)$ and width $(W)$ dimensions. This rectangular area is divided into vertical sections (bays) to allocate a set of facilities. Bay width is determined using the area required for facilities that integrate this bay and the height of the plant. Each of these bays is subdivided to allocate the facilities that make up the layout. The bays are flexible in the sense that their widths will adapt to the facilities that they contain (Tate and Smith, 1995).

In order to encode an FBS layout in a chromosome data structure, two vectors are used (Figure 2). The first vector represents a permutation of integers identifying the facilities (1 through $n$, where $n$ is the number of facilities). This sequence represents the facility sequence bay by bay, from left to right and from bottom to top. The second vector contains $n-1$ binary elements indicating where the bay

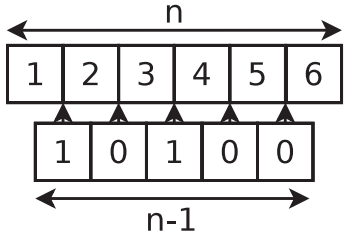

a) Two vector encoding

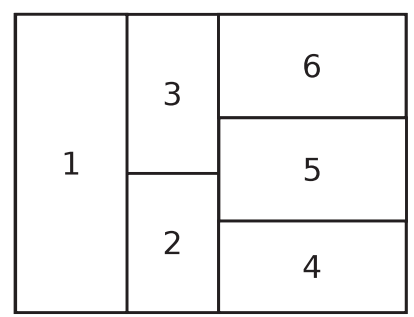

b) Corresponding layout with 3 bays and 6 facilities.
Figure 2: Two vector encoding for flexible bay structure. 
divisions are established in the plant layout. Therefore, when the value 1 appears, the facility that is in the same position in the first vector is the last element of the bay. Figure 2 shows an example of an encoding (of three bays and six facilities) and its corresponding layout.

\subsection{Selection, crossover and mutation operators}

The selection operator guides evolution with a probabilistic selection of the solutions that will make up the new offspring. The novel proposed MO-IGA applies two different selection operators. The initial GA uses tournament selection, as it considers one single objective (material flow). The interactive part uses the selection strategy of NSGA2 (Deb et al., 2000), which is multiobjective (subjective DM evaluation and material flow).

The crossover operator mates two individuals (Holland, 1992). In the proposed encoding, there are two vectors of a different nature: an ordered sequence (facilities) and a binary string (cutting points). Partially matched crossover operator is applied to the sequence of facilities and onepoint crossover to the binary vector representing the cutting points. Both operators are applied simultaneously.

The mutation operator changes one of the vectors chosen with half probability. In the facility sequence, mutation swaps the position of two facilities. In cutting points, one of the following two operations is selected with half probability: flip one binary value, merging two bays if the value was one or splitting them if the value was zero; or shift one randomly chosen cutting point to the left or right (each with half probability).

\subsection{Facility layout evaluation}

The proposed MO-IGA evaluates three main features in a facility layout design. First of all, the material flow is evaluated as defined in Section 4. Secondly, a DM subjective evaluation is assigned to each layout. In order to avoid DM fatigue, only a few layouts are evaluated, and their evaluation is then generalized to the other solutions. This evaluation process is described in detail in Section 1. Finally, aspect ratio is considered. Rather than discarding infeasible solutions according to aspect ratio constraints, MO-IGA penalizes them with the adaptive penalty function proposed by Tate and Smith (1995). This penalization is applied over both objectives: material flow and subjective evaluation. In this way, some infeasible solutions are preserved in order to allow convergence of those solutions that fall somewhere between feasible and infeasible (Coello et al., 2007).

5.3.1. Subjective evaluation by the $D M$ This evaluation is purely subjective, based on the DM opinion at each generation. The DM assigns a mark to each facility layout evaluated. This mark can fluctuate from a minimum value of 1 (the DM does not like the solution shown) to a maximum value of 5 (the DM likes the displayed solution very much).

Visual information is a fundamental key in any interactive approach, and is required in order to pass the maximum quantity of information to the DM. The proposed MO-IGA shows the facility layout including material flow and aspect ratio constraint satisfaction, as explained in Section 2. Figures 4-14 are examples of this representation.

The evaluation of many facility layouts is a very tiring and demanding task. This may lead to mistakes or early stopping of the algorithm, worsening results in both cases. In order to avoid DM fatigue, the population is classified into clusters of similar individuals, after which only one representative element of each cluster is displayed to the DM. The proposed MO-IGA identifies nine clusters following those of Kamalian et al. (2004), which indicates that showing nine representative solutions to the DM is the most effective way. The evaluation of each representative is generalized to the rest of the individuals of the cluster.

In order to cluster the population individuals, phenotype features are extracted from each encoded individual (genotype). These features describe the physical facility layout generated; the idea is to find similarities among the solution layouts in the population. The proposed MO-IGA utilizes the coordinates of two points defining the rectangle (top left and bottom right corners) occupied for each facility.

Using these phenotype features, the fuzzy c-means clustering algorithm (Bezdek et al., 1984) is applied, as explained in Section 2. This is an overlapping method offering the possibility of an element being a member of different clusters while at the same time maintaining a certain degree of membership. Using the membership degree of the individual to each group, the subjective evaluation of the remaining individuals is calculated with equation (8).

$$
s . e_{j}=\sum_{i}^{c} m_{i j} e_{i}
$$

where s. $e_{j}$ is the estimated subjective evaluation of individual $j, c$ is the number of clusters, $m_{i j}$ is the membership grade of the individual $j$ in the cluster $i$ and $e_{i}$ is the mark assigned by the DM to the representative element of cluster $i$.

\section{Empirical evaluation}

The novel proposed MO-IGA has been applied on two realworld industrial UA-FLPs: an ovine slaughterhouse and a carton recycling plant.

\subsection{Parameter tunning}

The novel proposed MO-IGA has parameters that must be set up. They have been fitted running the MO-IGA over the slaughterhouse problem case (described in Section 2) to achieve good performance. The values chosen provide a good starting point for this interactive system and could be used directly by a designer without knowledge of evolutionary algorithms.

Table 1 shows the values tested for each parameter and the value chosen to be used in the comparisons. The experiments and the motivation of the choice for each parameter are as follows. 
Table 1: Proposed multi-objective interactive genetic algorithm parameter values

\begin{tabular}{lcl}
\hline Parameter & Value & \multicolumn{1}{c}{ Tested values } \\
\hline Number of initial generations & 200 & $50,100,200,500$ \\
Population size & 500 & $100,200,500$, \\
& & 1000 \\
Crossover probability & 0.7 & $0.5,0.7,0.9$ \\
Mutation probability & 0.3 & $0.1,0.2,0.3$ \\
Tournament size & 2 & $2,3,4$ \\
Number of interactive & 100 & $25,50,100$ \\
generations & & 7,9 \\
Number of clusters & 9 & $1.025,1.05,1.1$ \\
Fuzziness in c-means & 1.025 & \\
\hline
\end{tabular}

The number of initial generations influences the quality of the population to be used as input of the interactive phase. This parameter is chosen to provide a diverse population, fitted to low material flow and rich on feasible layouts (according to aspect ratio constraints). A good balance is provided when the initial GA runs for 200 generations; a higher value degenerates in a population very fitted to material flow but with reduced diversity to allow DM choices. Standard deviation of population material flow fitness with aspect ratio penalty is used to detect diversity loss. In this case, the choice of this parameter can probably be automated to fit each problem and deserves more specific research.

The number of individuals in the population was increased to 500 in order to have a great diversity of solutions. This allows the initial population on the MOIGA to be very rich in plants fitting material flow and aspect ratio. In this way, when the DM starts evaluating and plants are discarded during the selection process for not meeting DM demands, there is still a large pool of good solutions. Lower values gave poorer results, and higher values did not improve the process.

After tests with all valid combinations of the values indicated in Table 1, tournament size, crossover and mutation probability were chosen to allow for a good balance between exploration and exploitation of the search space.

The number of generations in the interactive phase is decided by the DM on line, stopping when satisfactory solutions are found. In the tests conducted under this research, we have tried to keep this number as low as possible, finally setting it to 100 , in order to reach the best results. This value is feasible but a bit large for an expert evaluating. However, a good final layout design deserves the effort. Further improvements in the unsupervised learning included or in the evolution process are interesting for reducing $\mathrm{DM}$ fatigue. In this sense, the proposal described in this study opens new fields of research.

The number of clusters are determined by the number of representative solutions to evaluate. Using nine representative solutions is suggested in literature (Kamalian et al., 2004) as explained in Section 1. We consulted the DM as to whether he preferred seeing nine solutions or only seven.

Fuzziness in c-means regulates the degree in which each item belongs to several clusters, from 1, when each item would belong to only one cluster, to greater values where belongingness is spread among the clusters.
Although the novel proposed MO-IGA is the first proposal that combines an interactive evaluation with the optimization of material flow in UA-FLP, only the GA of Enea et al. (2005) and the IGA of García-Hernández et al. (2013) were used to compare the material flow results with other proposals. The parameters of the IGA have been set to the same values as in MO-IGA to perform a fair comparison. The parameter values of Enea's GA are taken from Enea et al. (2005) and are modified to obtain better results with the cases used, except population size and the number of generations. The population size is set to be equal to that of the MO-IGA, while the number of generations is set to a higher value. Table 2 lists the parameter values used.

\subsection{Ovine slaughterhouse problem case}

6.2.1. Description An ovine slaughterhouse projected in Córdoba (Spain) is analysed in this first problem case (Salas-Morera et al., 1996). The available space is a rectangular area of $30 \mathrm{~m} \times 51.14 \mathrm{~m}$, fitting the space needed for all the facilities $\left(1534 \mathrm{~m}^{2}\right)$. Table 3 lists the facilities with their required area and maximum acceptable aspect ratio. Figure 3 illustrates the material flow in this process. It starts from facility A to facility B. There, the material flow splits into three paths: B-E-F-J $(60 \%$ of material), B-C-G-L $(15 \%)$, B-D-L $(10 \%)$. Facility B process discards the remaining $5 \%$ of material.

Table 2: Parameter values used for Enea's GA (Enea et al., 2005)

\begin{tabular}{lc}
\hline Parameter & Value \\
\hline Number of generations & 1000 \\
Population size & 500 \\
Maximum number of clones & 10 \\
Severity parameter k for penalty of unfeasible solutions & 3 \\
Search probability & 0.5 \\
Number of iterations to perform search for all & 500 \\
population & \\
Crossover probability & 0.7 \\
Mutation 1 probability & 0.3 \\
Mutation 2 probability & 0.2 \\
Initial bay size & 7 \\
\hline
\end{tabular}

Table 3: Ovine slaughterhouse facilities

\begin{tabular}{llcc}
\hline Id & \multicolumn{1}{c}{ Facility } & Area $\left(\mathrm{m}^{2}\right)$ & Aspect ratio limit \\
\hline A & Stables & 570 & 4 \\
B & Slaughter & 206 & 4 \\
C & Entrails & 150 & 4 \\
D & Leather and skin & 55 & 4 \\
E & Aeration chamber & 114 & 4 \\
F & Refrigeration chamber & 102 & 4 \\
G & Entrails chamber & 36 & 4 \\
H & Boiler room & 26 & 4 \\
I & Compressor room & 46 & 4 \\
J & Shipping & 109 & 4 \\
K & Offices & 80 & 4 \\
L & Byproduct shipping & 40 & 4 \\
\hline
\end{tabular}




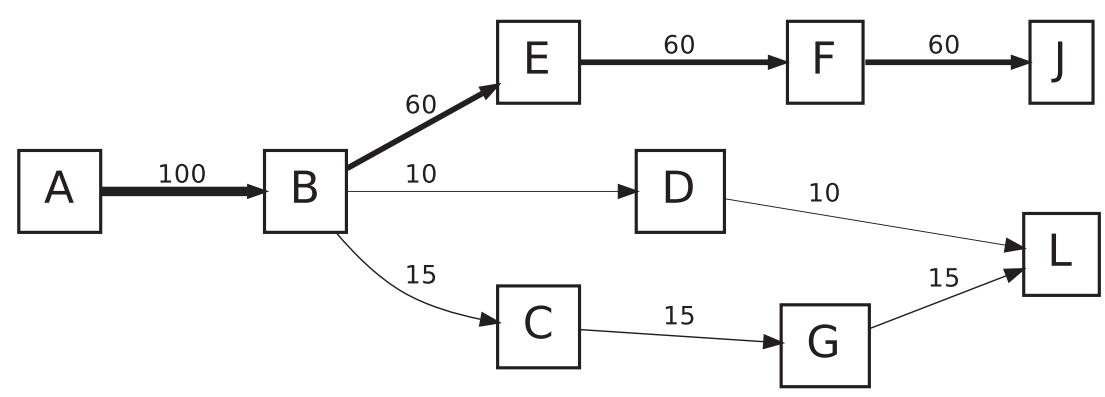

Figure 3: Material flow in ovine slaughterhouse.

The considered UA-FLP lacks explicit qualitative requirements in its definition. However, the real design must establish other implicit requirements. A DM on the field of these facility layout designs has assisted the development and evaluation of our novel approach. Table 4 shows qualitative interests identified by the DM while using the MO-IGA. Giving one point for the accomplishment of each criterion, the DM evaluation is simulated to allow performing tests and reproducibility of results.

Note that the types of the criteria identified are specific to this problem. They have been identified after working directly with the DM. However, in practical situations, it may be hard to extract these requirements before and during the layout design process. The goal of this simulated evaluation is to provide a real example of DM demands to test the proposal. Obviously, if modelling all possible types of designer demands in this way were possible, our proposal would be useless.

6.2.2. Results Running the novel proposed MO-IGA on the ovine slaughterhouse problem returned the pareto front with 11 solutions summarized in Table 5 . These pareto front tables are ordered in descending order of the DM evaluation, which gives ascending order of preferable material flow (to be minimized). The best value for each objective is shown in bold face. The DM evaluations are real values due to the clustering generalization of DM integer evaluations of similar plants.

Figures 4-8 represent the most interesting solutions selected by the expert from the pareto front of Table 5 . Figures representing the facility layout designs show the facility placement and illustrate the following layout properties:

1. The material flow is represented with an orange line of variable width. The line goes straight between the facility centres, which allows a lower superposition of

Table 4: Decision maker desires over the facility layout of the ovine slaughterhouse

\begin{tabular}{|c|c|c|}
\hline Id & Facilities & Criterion \\
\hline 1 & A & To be at one end of the plant (east or west) \\
\hline 2 & $\mathrm{~J}, \mathrm{~K}$ & $\begin{array}{l}\text { To be on the perimeter of the plant (next } \\
\text { to the outside) and apart from each other }\end{array}$ \\
\hline 3 & A & To be apart from $\mathrm{E}$ and $\mathrm{F}$ \\
\hline 4 & $\mathrm{~A}, \mathrm{C}, \mathrm{D}, \mathrm{I}, \mathrm{G}$ & To be apart from $\mathbf{J}$ and $\mathrm{K}$ \\
\hline
\end{tabular}

Table 5: Multi-objective interactive genetic algorithm final pareto front of facility layouts for the ovine slaughterhouse

\begin{tabular}{lccc}
\hline Number & $\begin{array}{c}\text { Material } \\
\text { flow }\end{array}$ & $\begin{array}{c}\text { Decision maker } \\
\text { evaluation }\end{array}$ & Graphic \\
\hline 1 & 5325 & 5.00 & Figure 4 \\
2 & 5220 & 4.41 & Figure 5 \\
3 & $\dagger 4777$ & 4.40 & Figure 6 \\
4 & 4703 & 4.29 & Figure 7 \\
5 & 3920 & 4.24 & \\
6 & 3749 & 4.23 & \\
7 & 3688 & 4.21 & \\
8 & 3651 & 4.18 & Figure 8 \\
9 & 3635 & 3.06 & \\
10 & 3625 & 3.99 & \\
11 & $\mathbf{3 6 0 6}$ & & \\
\hline
\end{tabular}

The best material flow and decision maker evaluation compromise according to expert.

lines than using paths allowing a better identification of material flow.

2. If an aspect ratio constraint is not satisfied, the facility background is filled with red colour.

3. A red line between a pair of facilities indicates a violation of the undesired adjacency criterion identified by the DM between these two facilities.

As seen in Figures 4-8, all offered plant layouts satisfy aspect ratio constraints. Because of penalization, only a few unfeasible plants were shown to the DM during the evolution. They have not been filtered to allow the DM to evaluate other aspects shown in these plants. However, they are automatically penalized and quickly discarded, and in this way, they are absent in the final pareto front. All final designs proposed to the DM satisfy criteria 1, 2 and 3. Criterion number 4 is harder to achieve (as it requires more conditions than the other criteria), and most solutions satisfy it only partially.

The first solution (Figure 4) fully satisfies all DM criteria. However, there are better plant layouts in terms of material flow. The second solution (Figure 5) improves on material flow at the cost of reducing DM satisfaction. The third (Figure 6) and fourth solutions follow the same tendency. However, the fifth solution (Figure 7) shows a very interesting design for the DM because it considerably reduces the material flow, while subjective DM evaluation is almost perfect (only facilities I and $\mathbf{J}$ are adjacent and not very much). The fact that this solution was considered worse than the previous ones by the algorithm is due to the generalization error when 


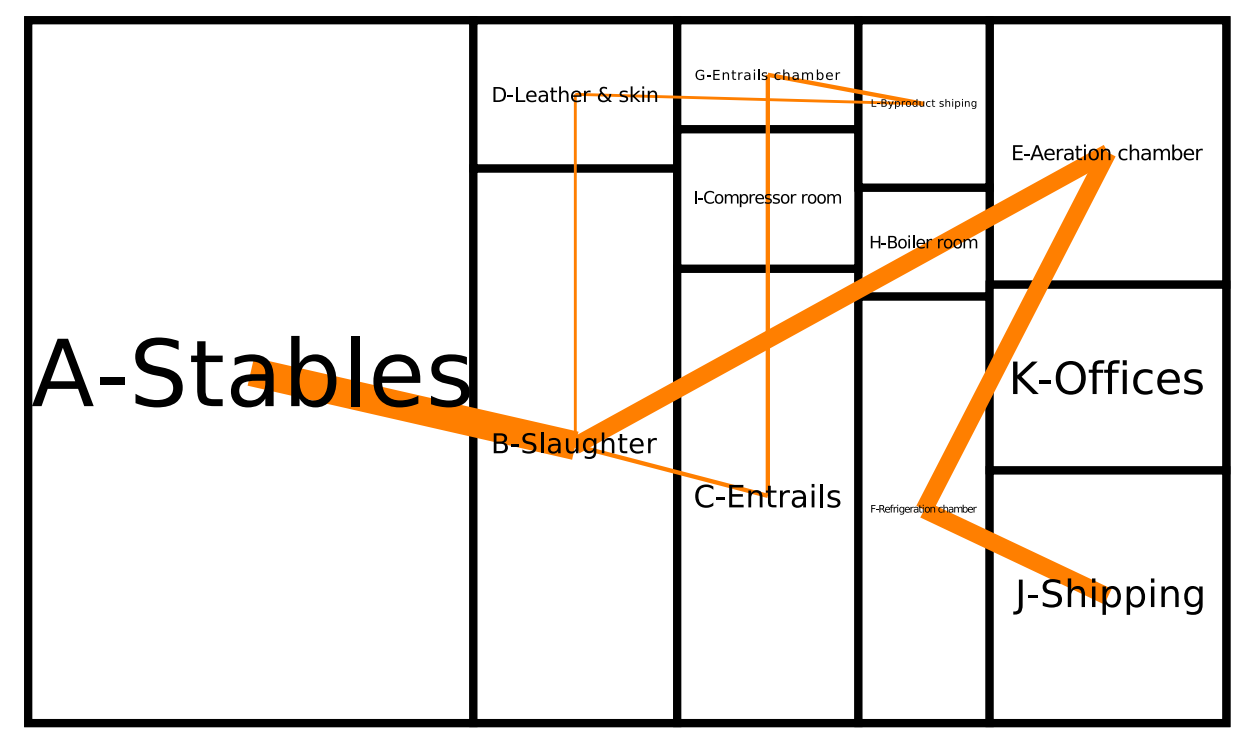

Figure 4: Multi-objective interactive genetic algorithm's first slaughterhouse solution.

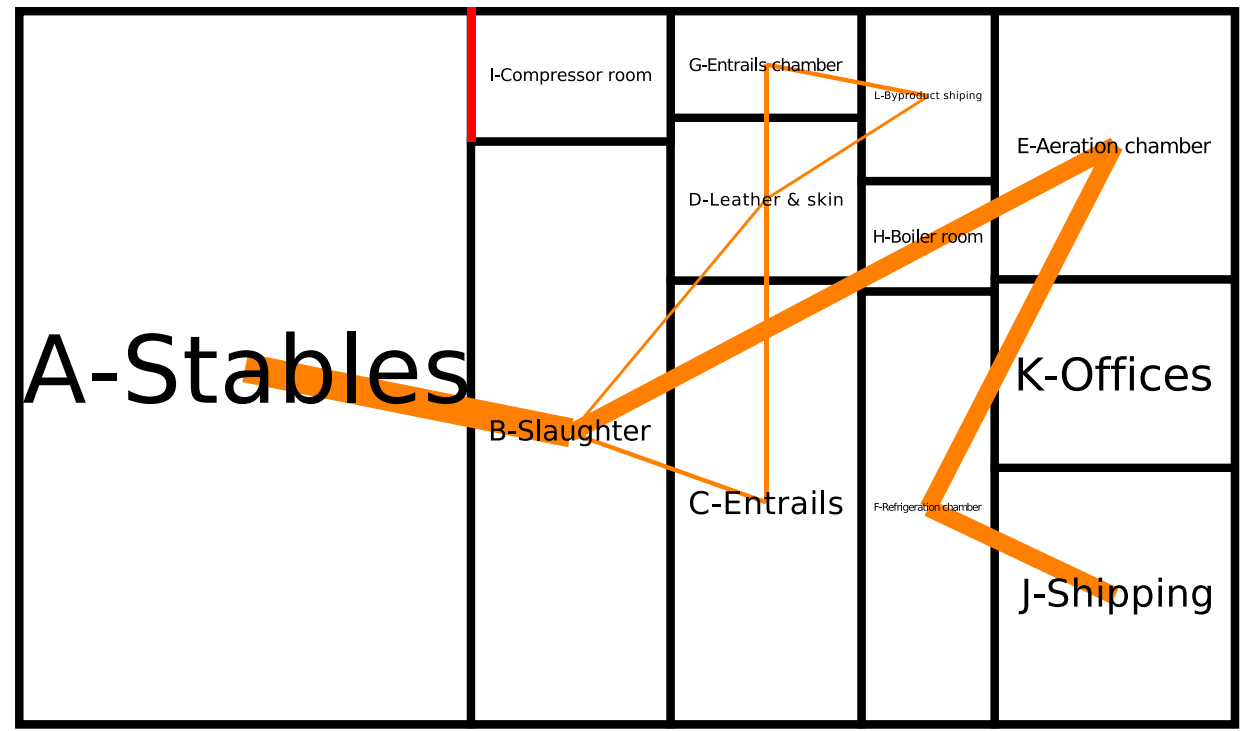

Figure 5: Multi-objective interactive genetic algorithm's second slaughterhouse solution.

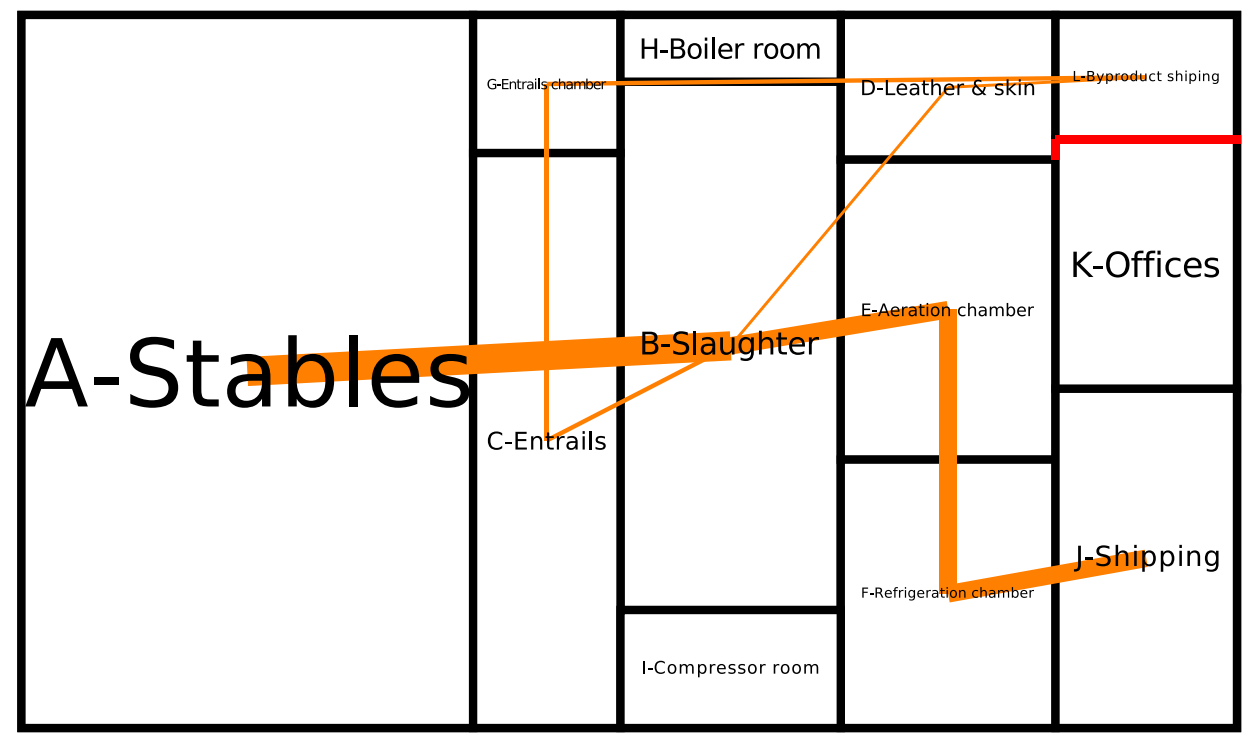

Figure 6: Multi-objective interactive genetic algorithm's third slaughterhouse solution. 


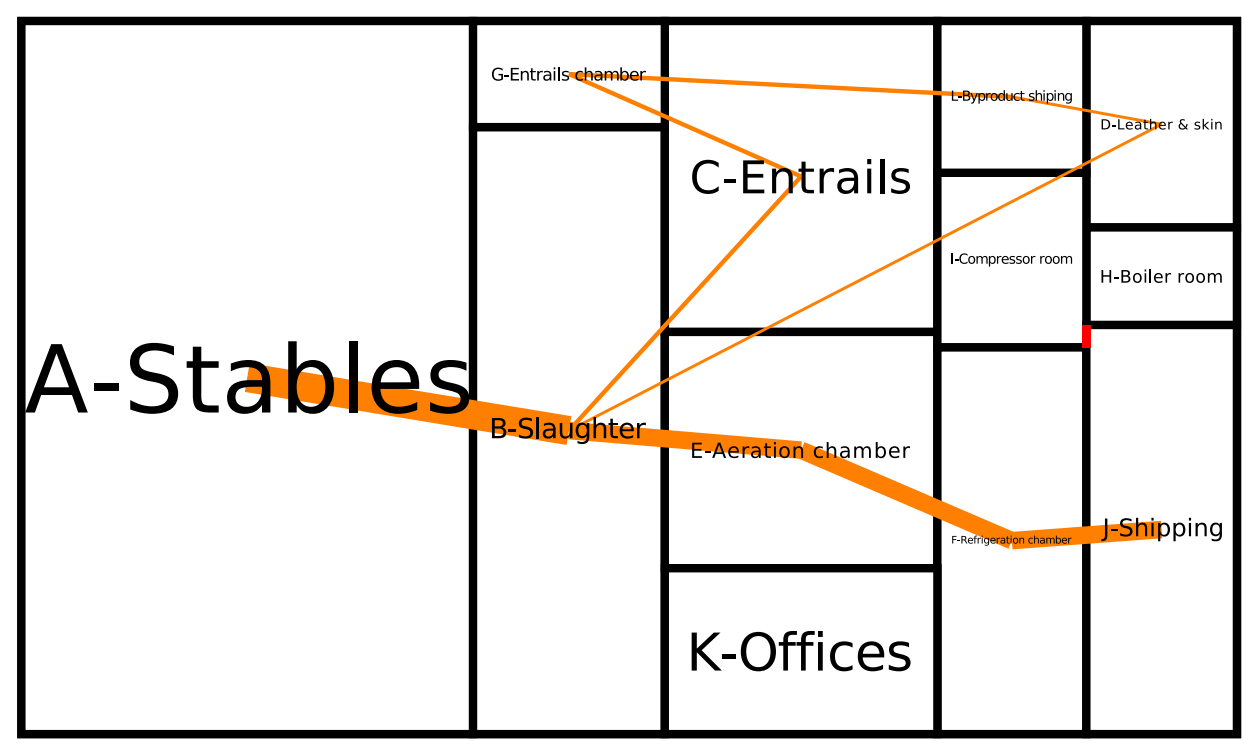

Figure 7: Multi-objective interactive genetic algorithm's fifth slaughterhouse solution.

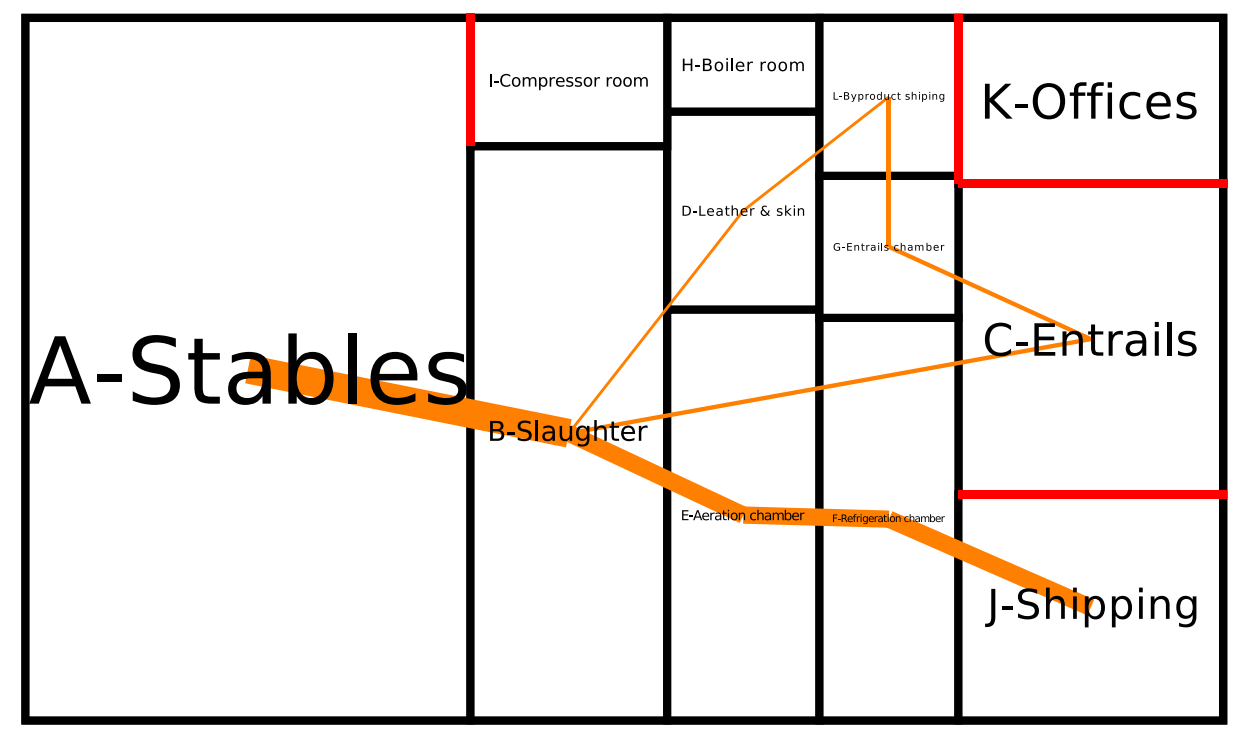

Figure 8: Multi-objective interactive genetic algorithm's 11th slaughterhouse solution.

using clustering to extrapolate evaluations. The error is small (relative to the evaluation range), and it has not prevented the algorithm from finding this good solution. Nevertheless, finding a way to reduce generalization error on DM evaluation can improve the evolution, and by extension the results, by avoiding the loss of good plants. The tendency of the following solutions in the pareto front is improving the material flow at the cost of worsening the DM evaluation. Figure 8 shows the last solution, where material flow is the lowest at the cost of being the worst in satisfying DM criteria.

For the purposes of comparison, the IGA alone has been applied, together with Enea's GA as a benchmark model. Both algorithms are run the same number of times as there are solutions present in the pareto front obtained with MO-IGA for each problem. In this way, the same number of solutions are presented to the DM, who decides if using the multi-objective interactive approach is worth the effort. Table 6 shows all the results by using Enea's GA. Figure 9 shows the best solution for the DM. There were other solutions with a lower material flow of value 3854 but with a worse distribution from the DM point of view (shipping

Table 6: Enea's best facility layout for each run on ovine slaughterhouse problem

\begin{tabular}{lcc}
\hline Number & Material flow & Graphic \\
\hline 1 & 4436 & \\
2 & 4819 & \\
3 & 8621 & \\
4 & 7114 & Figure 9 \\
5 & 4218 & \\
6 & $\dagger 5772$ & \\
7 & 6344 & \\
8 & $\mathbf{3 8 5 4}$ & \\
9 & 5516 & \\
10 & 7031 & \\
11 & 4218 & \\
\hline
\end{tabular}

The best according to expert. 


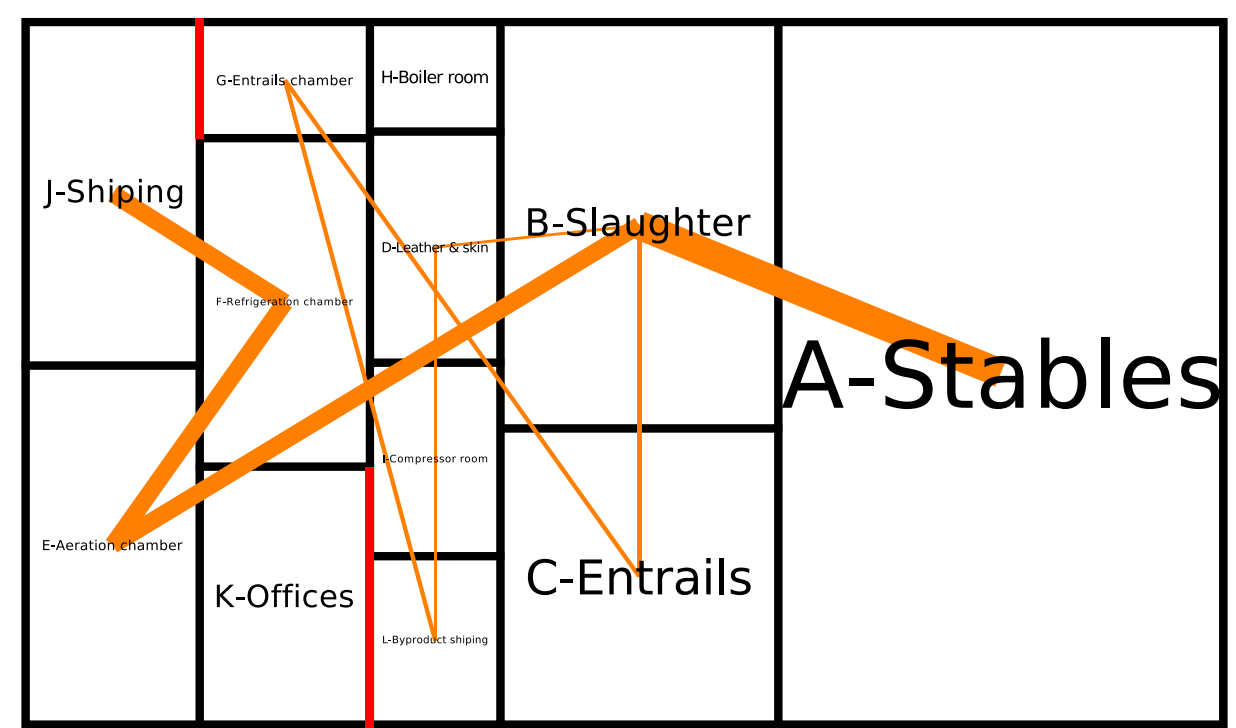

Figure 9: Sixth solution found by Enea's genetic algorithm for slaughterhouse.

not on the outside, with undesired adjacent facilities). Comparing the results with the proposed MO-IGA, the DM criteria are best fitted with our novel MO-IGA. This was expected as MO-IGA is the first proposal designed to include subjective knowledge in UA-FLP together with material flow optimization, while Enea's GA does not consider subjective criteria. Regarding material flow optimization, the proposed MO-IGA achieves results comparable to those of Enea's GA. Table 7 shows the results of the IGA. In this case, IGA achieves good DM evaluation and good material flow on some runs. However, as the material flow is ignored, most of the runs proposed worse solutions. It is important to keep in mind that each time this algorithm is run, the DM must be asked the same number of times that MO-IGA to obtain the whole pareto front. In this way, running the MO-IGA is less costly.

\subsection{Carton recycling plant problem case}

6.3.1. Description In this second real case, the DM wants to design a layout to be used in a project for the development of a carton recycling plant. As with the slaughterhouse problem, Table 8 and Figure 10 define this design as an UA-FLP instance.

Table 7: Interactive genetic algorithm's best facility layout for each run on ovine slaughterhouse problem

\begin{tabular}{lcc}
\hline Number & Material flow & Decision maker evaluation \\
\hline 1 & 6488 & 4.00 \\
2 & 7126 & 4.00 \\
3 & 8456 & 4.00 \\
4 & $\mathbf{4 5 7 8}$ & $\mathbf{5 . 0 0}$ \\
5 & 6210 & 5.00 \\
6 & 6247 & 5.00 \\
7 & 7536 & 5.00 \\
8 & 4999 & 5.00 \\
9 & 5840 & 5.00 \\
10 & 6898 & 5.00 \\
11 & 7112 & 4.00 \\
\hline
\end{tabular}

After preliminary use of the novel MO-IGA, the DM has realized the requirements of this facility layout, apart from the material flow relations. As presented in Table 9, each row criterion is assigned one point to simulate the DM evaluation (with the same purpose as the evaluation described for the slaughter house design).

6.3.2. Results Table 10 shows the resulting pareto front after applying the novel proposed MO-IGA on the carton recycling plant problem. The most representative results are illustrated in Figures 11-13. These figures include straight lines between the centre of two facilities indicating the DM desire to have the two facilities near to each other. The line is coloured with a gradient from green (near) to red (far), expressing the degree to which they are satisfying the criterion.

The first facility layout design obtained (Figure 11) fits all DM criteria perfectly. While improving material flow, the third solution (Figure 12) still satisfies the DM criteria quite well. The last solution (Figure 13) has the best material flow but fails to satisfy DM criteria. The Enea's GA solutions (Table 11) include the worst solutions optimizing the material flow and the best solution selected by the DM (Figure 14), only satisfying some of the criteria. Table 12 shows the results of the IGA. This algorithm

Table 8: Carton plant facilities

\begin{tabular}{llcc}
\hline Id & \multicolumn{1}{c}{ Facility } & Area $\left(\mathrm{m}^{2}\right)$ & Aspect ratio limit \\
\hline A & Raw material & 40 & 4 \\
B & Finished product & 40 & 4 \\
C & Mechanic & 20 & 4 \\
D & Offices & 50 & 4 \\
E & Staff WC & 20 & 4 \\
F & Expedition & 40 & 4 \\
G & Hydraulic 1 & 20 & 4 \\
H & Hydraulic 2 & 20 & 4 \\
I & Crushing & 20 & 4 \\
J & Circ. saw & 10 & 4 \\
K & Heat exchange & 10 & 4 \\
\hline
\end{tabular}




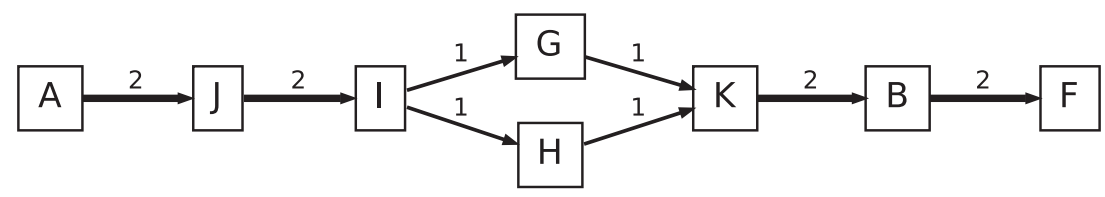

Figure 10: Material flow in carton plant.

Table 9: Decision maker desires over the facility layout of the carton plant

\begin{tabular}{lcl}
\hline Id & Facilities & \multicolumn{1}{c}{ Criterion } \\
\hline 1 & A & $\begin{array}{l}\text { To be on the perimeter } \\
\text { of the plant (next to the outside) } \\
\text { To be on the perimeter of the } \\
\text { plant (next to the outside) }\end{array}$ \\
2 & F & $\begin{array}{l}\text { To be near of E, F and A } \\
\text { To be near of J, I, G and H }\end{array}$ \\
3 & D & C
\end{tabular}

Table 10: Multi-objective interactive genetic algorithm final pareto front of facility layouts for the carton plant

\begin{tabular}{lccc}
\hline Number & $\begin{array}{c}\text { Material } \\
\text { flow }\end{array}$ & $\begin{array}{c}\text { Decision maker } \\
\text { evaluation }\end{array}$ & Graphic \\
\hline 1 & $\dagger 86.92$ & 5.00 & Figure 11 \\
2 & 81.99 & 4.32 & Figure 12 \\
3 & 80.02 & 4.28 & \\
4 & 78.10 & 4.28 & \\
5 & 77.23 & 4.23 & Figure 13 \\
6 & 73.97 & 4.13 & 4.12 \\
7 & 68.71 & 3.59 & \\
8 & 6.55 & &
\end{tabular}

${ }^{\dagger}$ The best material flow and decision maker evaluation compromise according to expert.

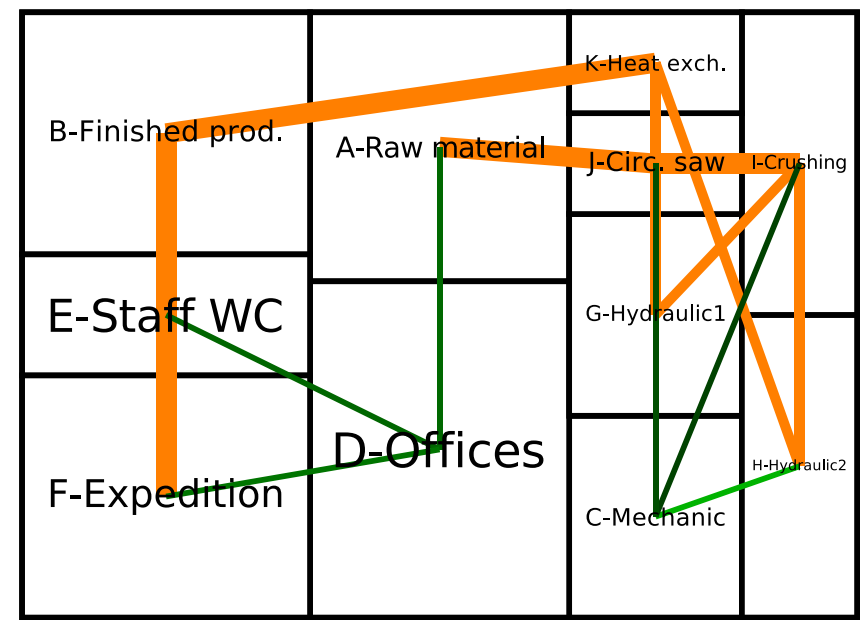

Figure 11: Multi-objective interactive genetic algorithm's first carton plant solution.

achieves good DM evaluation. However, as the material flow is ignored, the solutions obtained are worse than those reached by the MO-IGA.

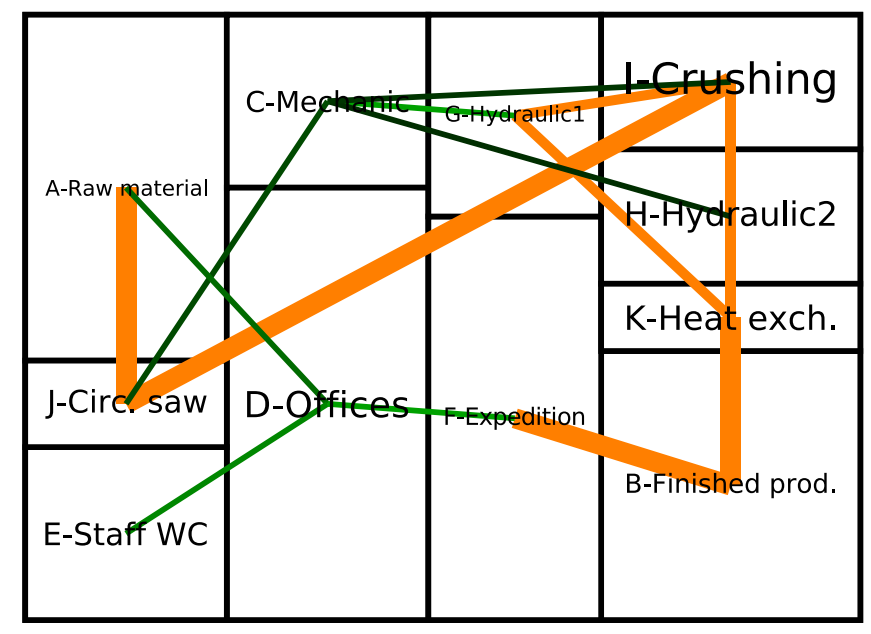

Figure 12: Multi-objective interactive genetic algorithm's third carton plant solution.

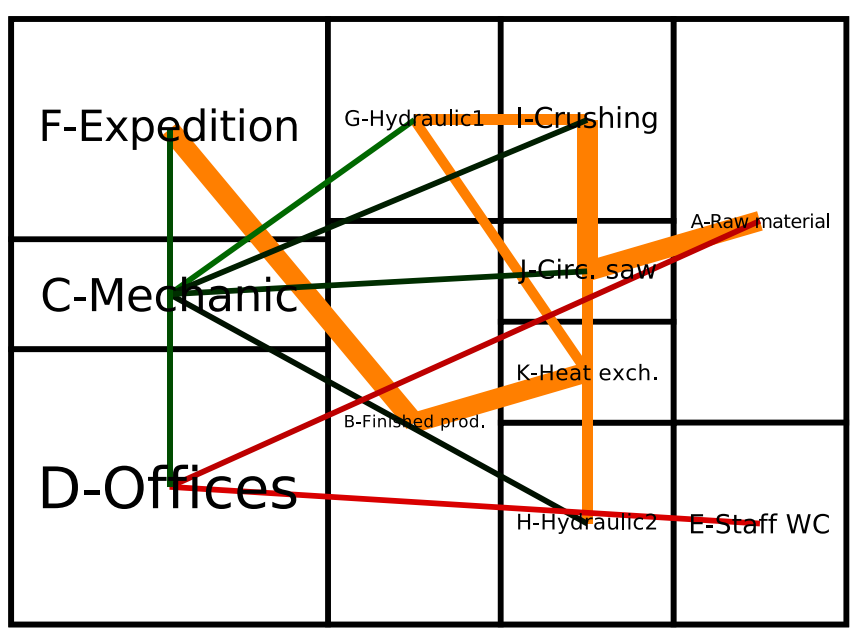

Figure 13: Multi-objective interactive genetic algorithm's eighth carton plant solution.

Table 11: Enea's best facility layout for each run on carton plant problem

\begin{tabular}{lrr}
\hline Number & Material flow & Graphic \\
\hline 1 & 103.02 & \\
2 & 102.82 & \\
3 & 110.57 & Figure 14 \\
4 & $1 \dagger 94.10$ & \\
5 & 129.58 & \\
6 & 115.93 & \\
7 & 99.02 & \\
8 & 111.11 & \\
\hline
\end{tabular}

${ }^{\dagger}$ The best according to expert. 


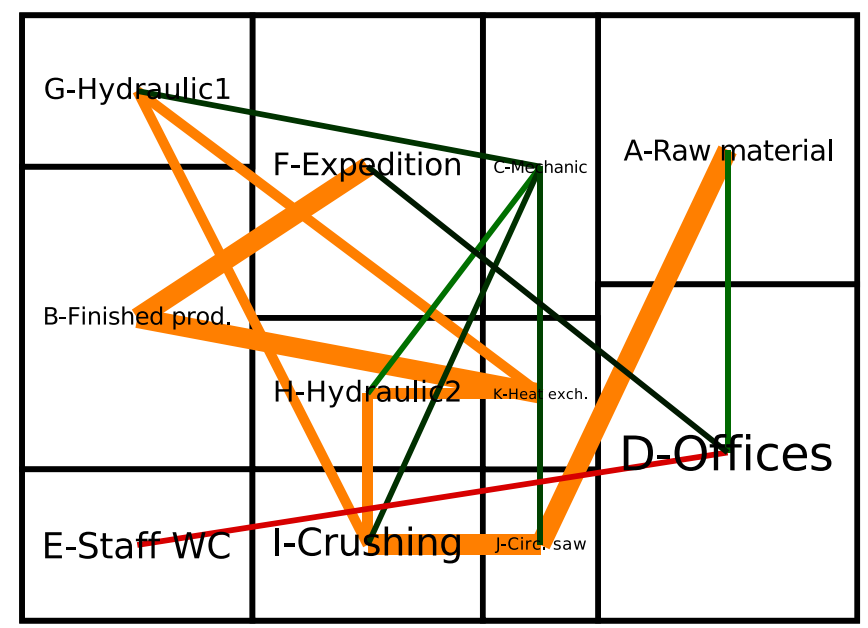

Figure 14: Fourth solution found by Enea's genetic algorithm for carton.

Table 12: Interactive genetic algorithm's best facility layout for each run on carton plant problem

\begin{tabular}{lcc}
\hline Number & Material flow & Decision maker evaluation \\
\hline 1 & 114.05 & 5.00 \\
2 & 145.75 & 5.00 \\
3 & 110.57 & 5.00 \\
4 & 115.75 & 5.00 \\
5 & 125.18 & 5.00 \\
6 & 141.60 & 5.00 \\
7 & 117.18 & 5.00 \\
8 & 142.96 & 5.00 \\
\hline
\end{tabular}

\section{Conclusions}

This study presents a novel MO-IGA for the UA-FLP. This approach allows taking the DM's expertise into account for the algorithm, guiding the search process and adjusting it to the DM preferences, while optimizing the material flow as in classical approaches.

In order to avoid overburdening, the DM with many requests of evaluation (all individuals that make up the population in each generation), a clustering method was used to group the population into similar clusters so that only the representative element of each cluster is shown to the DM per generation, and its evaluation is generalized to the rest of the population.

Our empirical studies show that the proposed MO-IGA is capable of capturing the aspects that the DM preferences. Furthermore, good solutions were obtained in a reasonable number of iterations.

In order to avoid DM fatigue, different techniques to reduce the number of human evaluations will be investigated in future work. Because many features from the solutions have to be considered, the DM could end up distracted. In this respect, this study opens the field to new research lines in the application of learning models, for example, improving clustering to reduce the generalization error in the evaluation phase, or including some supervised learning model to predict DM evaluation on unseen layouts (from previously evaluated designs), which will certainly improve results and reduce DM fatigue.
Additionally, another research line is the automation of the selection of the proposed MO-IGA parameters to better suit problems of any type.

\section{Acknowledgements}

This research is partially supported through projects of the Spanish Ministry of Economy and Competitiveness with reference TIN2010-21272-C02-01 (funded by the European Regional Development Fund) and SA405A12-2 from Junta de Castilla y León.

\section{References}

Aiello, G., M. Enea and G. Galante (2006) A multi-objective approach to facility layout problem by genetic search algorithm and electre method. Robotics and Computer-Integrated Manufacturing, 22, 447-455.

Aiello, G., G.L. Scalia and M. Enea (2012) A multi objective genetic algorithm for the facility layout problem based upon slicing structure encoding. Expert Systems with Applications, 39(12), 10352-10358.

Armour, G.C. and E.S. Buffa (1963). A heuristic algorithm and simulation approach to relative location of facilities. Management Science, 9, 294-309.

Askin, R.G. and M. Mitwasi (1992). Integrating facility layout with process selection and capacity planning. European Journal of Operational Research, 57(2), 162-173.

Avigad, G. and A. Moshaiov (2009). Interactive evolutionary multiobjective search and optimization of set-based concepts. ansactions on Systems, Man, and Cybernetics--Part B, 39(4), 1013-1027.

BABBAR-SEBens, M. and B.S. Minsker (2012). Interactive genetic algorithm with mixed initiative interaction for multi-criteria ground water monitoring design. Applied Soft Computing, 12(1), $182-195$.

Baruque, B., Porras, S., and E. Corchado (2011). Hybrid classification ensemble using topology-preserving clustering. New Generation Computer, 29(3), 329-344.

Bezdek, J.C., Ehrlich, R., and W. Full (1984). FCM: the fuzzy c-means clustering algorithm. Computers and Geosciences, 10, 192-203.

Brintup, A.M., Ramsden, J., and A. Tiwari (2007). An interactive genetic algorithm-based framework for handling qualitative criteria in design optimization. Computers in Industry, 58, 279-291.

Chaudhry, S.S. (2006). A genetic algorithm approach to solving the anti-covering location problem. Expert Systems, 23(5), 251-257.

Coello, C.C., Lamont, G., and D. Van Veldhuizen (2007). Evolutionary Algorithms for Solving Multi-Objective Problems, 2nd edn. Genetic and Evolutionary Computation. Berlin, Heidelberg: Springer.

Corchado, E., Sedano, J., Curiel, L., and J.R. Villar (2012). Optimizing the operating conditions in a high precision industrial process using soft computing techniques. Expert Systems, 29(3), 276-299.

DAY, P. and A.K. NANDi (2011). Evolution of superfeatures through genetic programming. Expert Systems, 28(2), 167-184.

Deb, K., Agrawal, S., Pratap, A., and T. Meyarivan (2000). A fast elitist non-dominated sorting genetic algorithm for multi-objective optimisation: NSGA-II. In PPSN VI: Proceedings of the 6th International Conference on Parallel Problem Solving from Nature, London, UK: Springer-Verlag; 849-858.

Enea, M., Galante, G., and E. Panascia (2005). The facility layout problem approached using a fuzzy model and a genetic search. Journal of Intelligent Manufacturing, 16, 303-316.

GARCÍA-HERnÁNDEZ, L. (2011). Genetic approaches for the unequal area facility layout problem. PhD thesis.

García-Hernández, L., Araúzo-Azofra, A., Pierreval, H., and L. SAlas-Morera (2009). Encoding structures and operators 
used in facility layout problems with genetic algorithms. In ISDA '09: Proceedings of the 2009 Ninth International Conference on Intelligent Systems Design and Applications, Washington, DC, USA: IEEE Computer Society; pp 43-48.

García-Hernandez, L., Salas-Morera, L., and A. ArauzoAzofra (2011). An interactive genetic algorithm for the unequal area facility layout problem. In SOCO, pages 253-262.

García-Hernández, L., Pierreval, H., Salas-Morera, L., and A. Arauzo-Azofra (2013). Handling qualitative aspects in unequal area facility layout problem: an interactive genetic algorithm. Applied Soft Computing, 13(4), 1718-1727.

Gomez, A., Fernandez, Q., la Fuente Garcia, D.D., and P. GARCIA (2003). Using genetic algorithms to resolve layout problems in facilities where there are aisles. International Journal of Production Economics, 84(3), 271-282.

He, H. and Y. TAN (2012). A two-stage genetic algorithm for automatic clustering. Neurocomputing, 81(0), 49-59.

Holland, J.H. (1992). Adaptation in Natural and Artificial Systems. Cambridge, MA, USA: MIT Press.

JACKOWSKI, K. and M. WOZNIAK (2010). Method of classifier selection using the genetic approach. Expert Systems, 27(2), 114-128.

JAIN, A. (2010). Data clustering: 50 years beyond k-means. Pattern Recognition Letters, 31(8), 651-666.

JEONG, I. and K. KIM (2009). An interactive desirability function method to multiresponse optimization. European Journal of Operational Research, 195(2), 412-426.

Kamalian, R.R., Takagi, H., and A.M. Agogino (2004). Optimized design of mems by evolutionary multi-objective optimization with interactive evolutionary computation. In GECCO (2), pages 1030-1041.

KOMARUDIN and K.Y. Wong (2010). Applying ant system for solving unequal area facility layout problems. European Journal of Operational Research, 202(3), 730-746.

Konak, A., Kulturel-KonaK, S., Norman, B., and A. Smith (2006). A new mixed integer formulation for optimal facility layout. Operation Research Letters, 34(6), 660-672.

Kusiak, A. and S.S. Heragu (1987). The facility layout problem. European Journal of Operational Research, 29(3), 229-251.

LiU, Q. and R.D. Meller (2007). A sequence-pair representation and mip-model-based heuristic for the facility layout problem with rectangular departments. IIE Transactions, 39(4), 377-394.

Luque, M., Miettinen, K., Eskelinen, P., and F. Ruiz (2009). Incorporating preference information in interactive reference point methods for multiobjective optimization. Omega, 37(2), $450-462$.

MACQueEn, J. (1967). Some methods for classification and analysis of multivariate observations. In Proc. Fifth Berkeley Symp. on Math. Statist. and Prob., volume 1, pages 281-297. Univ. of Calif. Press.

Meller, R., Narayanan, V., and P. Vance (1999). Optimal facility layout design. Operations Research Letters, 23, 117-127.

Mitchell, M. (1998). An Introduction to Genetic Algorithms. Cambridge, MA, USA: MIT Press.
Norman, B.A. and A.E. Smith (2006). A continuous approach to considering uncertainty in facility design. Computers and Operations Research, 33(6), 1760-1775.

Raman, D., Nagalingam, S.V., and G.C.I. Lin (2009). Towards measuring the effectiveness of a facilities layout. Robot. Comput.-Integr. Manuf., 25(1), 191-203.

Salas-Morera, L., Cubero-Atienza, A.J., and R. Ayuso-Munoz (1996). Computer-aided plant layout. Informacion Tecnologica, 7(4), 39-46.

Sato, T. and M. Hagiwara (2001). Idset: interactive design system using evolutionary techniques. Computer-Aided Design, 33(5), 367-377.

Scholz, D., Petrick, A., and W. Domschke (2009). Stats: a slicing tree and tabu search based heuristic for the unequal area facility layout problem. European Journal of Operational Research, 197(1), 166-178.

Shariatzadeh, N., Sivard, G., and D. Chen (2012). Software evaluation criteria for rapid factory layout planning, design and simulation. Procedia $\{C I R P\}, 3(0), 299-304$.

Sherali, H., Fraticelli, B., and R. Meller (2003). Enhanced model formulations for optimal facility layout. Operations Research, 51, 629-644.

Singh, S. and R. Sharma (2006). A review of different approaches to the facility layout problems. The International Journal of Advanced Manufacturing Technology, 30, 425-433.

TAKAGI, H. (2001). Interactive evolutionary computation: fusion of the capabilities of EC optimization and human evaluation. Proceedings of the IEEE, 89(9), 1275-1296.

TAM, K.Y. (1992). A simulated annealing algorithm for allocating space to manufacturing cells. International Journal of Production Research, 30(1), 63-87.

TAte, D.M. and A.E. Smith (1995). Unequal area facility layout using genetic search. IIE Transactions, 27, 465-472.

Tompkins, J., White, J., Bozer, Y., and J. TAnchoco (2010). Facilities Planning, 4th edn. New York: Wiley.

Ulutas, B.H. and S. Kulturel-KonaK (2012). An artificial immune system based algorithm to solve unequal area facility layout problem. Expert Systems with Applications, 39(5), 5384-5395.

Umeda, Y., Takata, S., Kimura, F., Tomiyama, T., Sutherland, J.W., Kara, S., Herrmann, C., and J.R. Duflou (2012). Toward integrated product and process life cycle planning - an environmental perspective. Annals - Manufacturing Technology, 61(2), 681-702.

WANG, M.-J., HU, M.H., and M.-Y. KU (2005). A solution to the unequal area facilities layout problem by genetic algorithm. Computers in Industry, 56(2), 207-220.

WonG, K.-Y. and KomARUdin (2010). Solving facility layout problems using flexible bay structure representation and ant system algorithm. Expert Systems with Applications, 37(7), 5523-5527.

WU, Y. and E. Appleton (2002). The optimisation of block layout and aisle structure by a genetic algorithm. Computers and Industrial Engineering, 41(4), 371-387.

YEH, J.-Y. and T.-H. WU (2010). Cascade of genetic algorithm and decision tree for cancer classification on gene expression data. Expert Systems, 27(3), 201-218. 